\title{
Experiences from a community advisory Board in the Implementation of early access to ART for all in Eswatini: a qualitative study
}

Charmaine Khudzie Mlambo ${ }^{1 *}$ D , Eva Vernooij ${ }^{2}$, Roos Geut ${ }^{2}$ Eliane Vrolings ${ }^{3}$, Buyisile Shongwe ${ }^{4}$, Saima Jiwan ${ }^{5}$, Yvette Fleming ${ }^{3}$ and Gavin Khumalo ${ }^{4}$

\begin{abstract}
Background: Engaging communities in community-based health research is increasingly being adopted in lowand middle-income countries. The use of community advisory boards (CABs) is one method of practicing community involvement in health research. To date, few studies provide in-depth accounts of the strategies that $C A B$ members use to practice community engagement. We assessed the perspectives, experiences and practices of the first local CAB in Eswatini (formerly known as Swaziland), which was implemented as part of the MaxART Early Access to ART for All study.

Methods: Trained Swazi research assistants conducted two focus group discussions and 13 semi-structured interviews with CAB members who had been part of the MaxART study for at least 2.5 years. Interviews explored $C A B$ composition and recruitment, the activities of $C A B$ members, the mechanisms used to engage with communities and the challenges they faced in their role.

Results: The MaxART CAB played an active role in the implementation of the Early Access to Art for All study, and activities mainly focused on: (1) promoting ethical conduct, in particular privacy, consent and confidentiality; (2) communication and education, communicating about the study and educating the community on the benefits of HIV testing and early access to HIV treatment; and (3) liaising between the community and the research team. Strategies for interacting with communities were varied and included attending general community meetings, visiting health facilities and visiting public places such as cattle dipping tanks, buses, bars and churches. Differences in the approach to community engagement between $C A B$ members living in the study areas and those residing outside were identified.

Conclusion: The experiences of the first $C A B$ in Eswatini demonstrate that community engagement using $C A B s$ is a valuable mechanism for engaging communities in implementation studies. Considerations that could impact $C A B$ functioning include clearly defining the scope of the $C A B$, addressing issues of $C A B$ independence, the $C A B$ budget, providing emotional support for $C A B$ members, and providing continuous training and capacity building. These issues should be addressed during the early stages of CAB formation in order to optimize functioning.
\end{abstract}

Keywords: Community Advisory Board, CAB, HIV, Swaziland, Eswatini, Test and Treat, Early ART, Community Engagement, Community System Strengthening, Implementation research

\footnotetext{
* Correspondence: khudzie@gmail.com

${ }^{1}$ Clinton Health Access Initiative, Mbabane, Swaziland

Full list of author information is available at the end of the article
}

(c) The Author(s). 2019 Open Access This article is distributed under the terms of the Creative Commons Attribution 4.0 International License (http://creativecommons.org/licenses/by/4.0/), which permits unrestricted use, distribution, and reproduction in any medium, provided you give appropriate credit to the original author(s) and the source, provide a link to the Creative Commons license, and indicate if changes were made. The Creative Commons Public Domain Dedication waiver (http://creativecommons.org/publicdomain/zero/1.0/) applies to the data made available in this article, unless otherwise stated. 


\section{Background}

Community engagement, defined as the process of working collaboratively with groups of people affiliated by geographic proximity, special interest or similar situations, to address issues affecting the well-being of those people [1], has become increasingly common in global health research [2]. In community-based research, the rationale for community engagement is to promote ethical conduct by ensuring that research is relevant to the community where it is being conducted, and that local views are incorporated into the research process $[3,4]$. The specific benefits of engaging communities include protecting research participants by minimizing physical, psychosocial, social, economic and legal risks, strengthening the informed consent process by providing adequate information on research goals, risks and benefits $[5,6]$; showing respect by listening to and addressing community perspectives, promoting partnership between the community and researchers [4]; and strengthening the acceptability and quality of research [7]. Recognizing that the ethical implications of research can affect communities has opened up international debate on the values, goals and mechanisms of meaningfully involving communities in the research process $[4,8,9]$. These discussions have in turn resulted in several guiding documents and reports which promote good ethical practice [10-12].

Community engagement can take a number of forms, and one of these approaches is the establishment of a Community Advisory Board (CAB) or Community Advisory Group (CAG). A CAB is usually comprised of individuals who represent the community targeted for research and who serve as liaisons between the research team and the community. The specific role of a CABs, as documented in the literature, includes: acting as a link between researchers and the community by establishing trusting relationships between the research team and the community [13-16]; educating community members about the research [16]; representing community concerns and priorities to the research team; providing the research team with insights into the social and cultural context within which the community operates; assisting in the development and review of research protocols, study materials and informed consent tools; promoting recruitment procedures; and disseminating study findings [17].

While evidence in the literature details advantages of CABs in strengthening community engagement, some studies have documented challenges faced by CABs. Some of the challenges include limited understanding of health research among $C A B$ members [14]; inability to communicate scientific information and procedures [16]; monetary expectation and expectation of future employment [16]; dependence on research institutes or individual researchers for $\mathrm{CAB}$ finances and sustainability; a lack of authority to influence decisions concerning the research $[14,18]$ and a contradiction between the ethical mandate of the $C A B$ and the CAB's role in facilitating implementation of research [16]. These challenges often result in uncertainty about the advisory roles of $\mathrm{CAB}$ members and concerns that their involvement does not serve to adequately represent communities, but rather act as a superficial mechanism to adhere to donor requirements.

Two models of $\mathrm{CAB}$ composition that are commonly differentiated are the 'broad community' model that has representation from a cross-section of the community, and a 'population-specific' model that consists of the specific populations being targeted by the research project [19]. The term 'community' has varied definitions in the context of community-based research [20]. Community can to refer to: a group of people with diverse characteristics who are linked by social ties, share common perspectives and engage in joint action in geographical locations or settings [20]; a group of people with a common characteristic or illness; or a group of people residing within the immediate surroundings of a particular entity, e.g. a health facility [16]. Due to the lack of a homogeneous definition of the term 'community' in research, there are often contradictory assumptions amongst study team members about results and achievements of the contributions of a community in a particular study [20]. In the context of CABs, the lack of a standard definition of community brings forth the question of whether $C A B$ members can indeed represent an entire community, and who should represent community interests in community-based health research. In this study, the $C A B$ resembled the 'broad community model' as the $C A B$ members were selected to represent a broad representation of social groups in different geographical locations.

This article explores the experiences of the first local $\mathrm{CAB}$ in Eswatini, which was established as part of the MaxART Early Access to ART for All (EAAA) study. Despite the advantages of CABs, as illustrated in the literature, previous community-based research conducted in Eswatini had not included CABs as a mechanism for engaging communities. This paper highlights the experiences of the MaxART CAB members by exploring how they engaged communities, their approach to operationalizing $C A B$ roles, as well as the challenges and proposed recommendations, which could be valuable for the functioning of future $\mathrm{CABs}$ in the country and elsewhere.

\section{Methods}

\section{Study context}

The Kingdom of Eswatini is severely affected by HIV/ AIDS. HIV prevalence is $27 \%$ ( $>15$ years), while HIV 
incidence is $1.36 \%$, with approximately 7000 new cases annually [21]. As part of continued efforts to manage the HIV/AIDS epidemic, the Ministry of Health $(\mathrm{MOH})$ introduced the MaxART EAAA study as a pilot. The study was designed to evaluate the acceptability, affordability, feasibility and clinical outcomes of offering early access to ART to all people living with HIV (PLHIV), as well as to inform national decision-making with regards to future HIV program planning.

The MaxART study used a randomized steppedwedge design with control, transition and intervention phases implemented across 14 health facilities (and surrounding communities) in the Hhohho region. During the control phase, HIV positive clients were initiated on antiretroviral therapy (ART) following standard national treatment guidance (CD4 count of $<350$ cells $/ \mathrm{mm}^{3} /<500$ cells $/ \mathrm{mm}^{3}$ [from 2015], or WHO disease stage 3 or 4) [22]. During the transition and intervention phases, ART was offered regardless of CD4 count or disease stage.

At the same time, a qualitative sub-study was conducted aimed at understanding the role of the $C A B$ during the implementation of the MaxART study.

\section{CAB composition and functioning}

The $\mathrm{CAB}$ had 24 volunteer members, aged 24 to 68 years (19 female; five male). CAB members were selected to represent different demographic and social groups and socio-economic characteristics in the community. In the communities where the study was being conducted, individuals who were knowledgeable about health issues, in particular HIV/AIDS, or were either leaders in the community or were active in community groups were proposed as potential members of the $\mathrm{CAB}$.

Potential members were identified by community health committees, the $\mathrm{MOH}$ and the Swaziland $\mathrm{Na}$ tional Network of People living with HIV/AIDS (SWANNEPHA). All CAB members received invitation letters to join the $C A B$, and provided either written or verbal consent to participate. Thirteen members resided in the communities where the MaxART study was conducted and the remaining eleven resided outside the study areas. The $C A B$ represented various groups in the community, including PLHIV, youth, key populations traditional leaders, traditional healers, religious leaders, academia, nurses and community health workers.

A CAB secretary, employed by SWANNEPHA, facilitated the CAB's formation and administrative functioning and responsibilities, included scheduling $C A B$ meetings, taking meeting minutes, reporting and arranging $C A B$ transport logistics. At the CAB's inception, the MaxART research team provided trainings on the study protocol and field standard operating procedures, research ethics, the basics of HIV, the history and functions of $\mathrm{CABs}$ and interpersonal communication skills. Since this was the first CAB in the country, the research team also collaborated with the AIDS Rights Alliance of Southern Africa (ARASA) to conduct trainings on human rights. Six refresher trainings were provided throughout the 3 years of implementation. Other support provided included transport reimbursement for attending meetings, t-shirts and name badges (to facilitate identification during visits to study facilities) and transportation to facilities for site-visits.

Prior to the CAB's formation, the research team contacted other teams in the African region with experience working with CABs in order to learn from their experiences, which was used to draft a terms of reference (ToR). The ToR was later discussed and finalized by the $\mathrm{CAB}$ members during their initial orientation session.

The CAB's aim was to ensure that all aspects of the study were conducted in accordance with human rights and ethical conduct as stated in the study protocol. The intended role of the $\mathrm{CAB}$ as detailed in the final TOR included:

- providing substantive input into all aspects of the study

- serving as the voice of the community and study participants

- ensuring that the study meets local needs and contributes to improved outcomes for people living with HIV

- building community capacity in research

- properly communicating information on the study and liaising with the community

- providing the study team with recommendations on study implementation

- monitoring progress and ensuring dissemination of study findings and providing quarterly updates to the study investigators

- ensuring that the study is conducted in accordance with human rights and ethical standards

Initially, $\mathrm{CAB}$ members used the broad guidance detailed in the TOR to facilitate engagement with community leaders. Following observations made during the first two quarterly $C A B$ meetings, which showed varied interpretation of the CAB's scope and activities amongst $\mathrm{CAB}$ members, discussions were held between the study team and $C A B$ members regarding key messaging on the purpose of the study and the role of the CAB. These discussions resulted in $C A B$ refresher training sessions that focused on topics such as the CAB's role in the community, including guidance on key topics of discussion with community members that could provide insight to the study team on community concerns about the study. 


\section{Data collection}

Of the $24 \mathrm{CAB}$ members invited to participate in the FGDs, eight were unable to attend due to logistical reasons. Participants for the semi-structured interviews were selected from the list of $\mathrm{CAB}$ members based on their geographical location and gender. Data was collected through two focus group discussions (FGDs) and 13 semi-structured interviews with $C A B$ members. In total researchers interviewed 16/24 (67\%) CAB members and 10 of these respondents also participated in focus group discussions. The characteristics of the participants are detailed in Table 1. The FGDs covered a broad range of topics, including $\mathrm{CAB}$ membership and recruitment, core activities, community engagement processes and related challenges. Key issues raised in the FGDs were subsequently investigated in semi-structured individual interviews with $13 \mathrm{CAB}$ members. Informed consent procedures were followed for both research components, including signed informed consent for the semistructured interviews and verbal consent from the FGD participants.

Swazi research assistants conducted the interviews in either Siswati or in a mix of English and Siswati. All interviews were recorded with digital voice recorders. Afterwards, the interviews were transcribed word for word and translated into English by the research assistant. The Swaziland National Health Research Review Board granted ethical approval prior to the study's commencement (Ref: MH599C/IRB 0009688/NHRRB 027/16).

\section{Data analysis}

Data were coded manually and thematic content analysis was utilized to pinpoint the central themes. Preliminary codes were based on initial research questions, and other significant topics that were identified during the first review of the interview transcripts were then included. KM carried out line-by-line coding in collaboration with GK and BS, who also discussed preliminary conclusions.

\section{Results}

\section{Perceived role of the $C A B$}

The intended role of the CAB in the EAAA study, as stated in the study protocol, was to "ensure that all aspects of the study were conducted in accordance with human rights and ethical standards" [23]. This broad and ambitious task was further detailed in the $C A B$ TOR to include functions such as communicating information on the study, liaising with the community, serving as the voice of the community and providing the study team with recommendations on study implementation. In addition to the information in the TOR, the study team also utilized sessions during $C A B$ refresher trainings to re-cap on the intended roles.

$\mathrm{CAB}$ members interviewed in this study understood their role as fulfilling three main functions: (1) promoting

Table 1 Characteristics of CAB members interviewed for the study

\begin{tabular}{|c|c|c|c|c|c|c|}
\hline CAB Member Identification & Gender & Age & $\begin{array}{l}\text { Residing in study } \\
\text { area (Yes/No }\end{array}$ & $\begin{array}{l}\text { Participated } \\
\text { in FGD1 }\end{array}$ & $\begin{array}{l}\text { Participated } \\
\text { in FGD2 }\end{array}$ & $\begin{array}{l}\text { Participated in semi- } \\
\text { structured interviews }\end{array}$ \\
\hline CAB member $A$ & $\mathrm{~F}$ & 38 & Yes & $x$ & & \\
\hline CAB member B & $\mathrm{F}$ & 36 & Yes & $x$ & & $x$ \\
\hline CAB member $C$ & $\mathrm{~F}$ & 31 & Yes & $x$ & & $x$ \\
\hline CAB member D & $\mathrm{F}$ & 41 & No & $x$ & & $x$ \\
\hline CAB member $\mathrm{E}$ & M & 42 & Yes & $x$ & & $x$ \\
\hline CAB member $F$ & $\mathrm{~F}$ & 53 & Yes & $x$ & & $x$ \\
\hline CAB member $G$ & M & 31 & Yes & $x$ & & $x$ \\
\hline $\mathrm{CAB}$ member $\mathrm{H}$ & $\mathrm{F}$ & 31 & Yes & & $x$ & $x$ \\
\hline CAB member I & $\mathrm{F}$ & 33 & Yes & & $x$ & $x$ \\
\hline CAB member J & $\mathrm{F}$ & 38 & Yes & & $x$ & \\
\hline CAB member $\mathrm{K}$ & $\mathrm{F}$ & 56 & No & & $x$ & $x$ \\
\hline CAB member $L$ & $\mathrm{~F}$ & 59 & No & & $x$ & \\
\hline CAB member $M$ & $\mathrm{~F}$ & 29 & No & & $x$ & $x$ \\
\hline CAB member $\mathrm{N}$ & $\mathrm{F}$ & 28 & No & & $x$ & \\
\hline CAB member $\mathrm{O}$ & $\mathrm{F}$ & 39 & Yes & & $x$ & \\
\hline CAB member $\mathrm{P}$ & M & 58 & Yes & & $x$ & \\
\hline CAB member Q & $\mathrm{F}$ & 31 & Yes & & & $x$ \\
\hline CAB member $\mathrm{R}$ & M & 32 & No & & & $x$ \\
\hline CAB member $\mathrm{S}$ & $\mathrm{F}$ & 54 & No & & & $x$ \\
\hline
\end{tabular}


research-related ethical conduct, in particular privacy, consent and confidentiality to community members; (2) communicating about the study and educating on the benefits of HIV testing and early access to HIV treatment; and (3) liaising between the community and the study team. Although these roles mostly represented the broad guidance provided in the $C A B$ terms of reference, as well as the messages used by the study team during $C A B$ trainings, some variations were noted in the interviews with regards to details in the mechanisms employed by $C A B$ members and the interpretation of their role when engaging community members. These issues are discussed further below.

\section{Promoting ethical conduct}

Nearly all CAB members mentioned their main role was to ensure that the human rights of community members were protected during the research process. In line with the guidance from the study team, the CAB's interpretation of this role included examining issues of privacy, consent and confidentiality for HIV positive study participants; enquiring whether study participants were informed of the study prior to enrollment and that informed consent was provided; enquiring into issues of coercion/forced ART initiation; observing that quality health services were being provided in the health facilities; and observing health worker well-being and working conditions in facilities. Topics discussed with clients and health workers in relation to this role included how the MaxART study was communicated to study participants, the relationship between health workers and the study team and adherence to informed consent procedures.

In addition to providing operational guidance on promoting human rights and ethical standards, the study team also recommended ways in which $C A B$ members could engage with the community as they executed their role. The proposed ways in which $\mathrm{CAB}$ members interacted with the community were varied, but mainly constituted observing and listening, as well as talking to community members. The $\mathrm{CAB}$ members who were residing in the study communities were advised to informally interact with community members in order to gauge perceptions about the study. As a result, observing the waiting areas of study facilities and listening to people were the main approaches to gather insight into the study implementation. $C A B$ members approached facility drop-ins and accompanied clients to educational health talks. Their methods also included joining patients in health facility queues, monitoring communication between health workers and clients and chatting informally with clients so as to document their attitudes towards and experiences of early ART initiation, the overall quality of service delivery at the facility and gaps in service delivery. In some instances, they disclosed their role as a CAB member to the clients. The following quote illustrates how these techniques of observing and listening were successful methods to gather unbiased information in a non-intrusive manner:

"Ok, my strategies which I thought worked better and
perfect don't come as a CAB member but as a
community member. Be a client also, come as a
human being, there is nothing different about you
anyways when you are a CAB member. So, when you
come, sometimes there is even no need to ask
questions, people will be talking about these issues
when standing in the queue waiting for a bus in the
morning, about EAAA or even ART. So try to probe
some few questions, try to engage like a client. Take
out your diary and start writing some few notes that
you have discovered. Or else as some point you can
even engage one client and explain to them that you
are a CAB member and I would love to engage you on
questions on the MaxART program that is going on
here and if you don't mind. If the client agrees to that,
you can proceed with asking." (CAB member 2).

When the $C A B$ was initially formed, $C A B$ members did not have any identifying clothing. Half way through the implementation of the MaxART study, as a result of recommendations from a case study on the functioning of the $C A B$ [24], $C A B$ members were provided with $t$ shirts and picture name tags to wear during health facility visits. The case study highlighted that $C A B$ members who did not disclose their role while actively seeking information from community members could potentially violate research ethics. $\mathrm{CAB}$ members were therefore advised by the study team to disclose to people their role as $C A B$ members during informal conversations.

For $\mathrm{CAB}$ members who did not reside in the study areas, visits to health facilities were part of prescheduled visits. These visits were usually attended by the $C A B$ secretary, the $C A B$ chair or co-chair and an additional $C A B$ member. For these visits, the $C A B$ secretary obtained permission from the facility management for $C A B$ members to engage with health workers and clients in order to understand how the study was being implemented and obtain information on concerns and recommendations.

\section{Communication and education}

According to the $\mathrm{CAB}$ TOR, another $\mathrm{CAB}$ role was communicating information about the purpose of the study, the benefits of early ART initiation, the study duration and the study implementation sites. In the interviews conducted with $\mathrm{CAB}$ members, it was evident that they fulfilled this role, including motivating people to 
participate in the study and debunking common myths about early ART use. They also expanded upon their initial role by including education on health issues outside the scope of the study.

$\mathrm{CAB}$ members advised people on general health issues and actively advocated for people in their communities to start HIV treatment early. This was partly related to the fact that some of the $C A B$ members, such as those who were nurses, rural health motivators and community health committee members, were knowledgeable about medical issues and had prior experience talking to people about health care issues. In addition, the trainings provided to the $C A B$ as part of the study included information on several health topics, including HIV/AIDS. Thus, $C A B$ members did not always differentiate between their role in the $C A B$ and their other healthrelated duties, but rather incorporated the $\mathrm{CAB}$ role and the study messages into their ongoing work.

In order to reach different members of the community, $C A B$ participants utilized existing social structures and networks. These included bars, community meetings, cattle dipping tanks, women's meetings, clinics, support group meetings for PLHIV, churches and public transportation. The community networks allowed for daily interaction with various community members, and since many of the $C A B$ members were already known in the communities, this facilitated acceptance and trust from community members, as illustrated in the following quote.

"I saw that it [the $C A B]$ is very important, because people in the community would listen to a person who stays within the community, compared to an outsider who just comes and goes. Only few will listen to a person from outside... whereas if someone from the same community says there is this program, it helps there and there, tries and explain to them, they will understand." (CAB member 10).

\section{Liaising between the community and the study team}

In addition to the facility visits and community interactions, another strategy that enabled the $C A B$ to address community concerns was the creation of a 24-h toll-free hotline. Previous studies had created hotlines for $C A B$ members [7], but in this study, the hotline was also accessible to community members. The hotline's purpose was to allow for quick reporting on study-related concerns or issues. The $C A B$ secretary ran the hotline, which was funded through the study budget at a cost of USD 76 monthly. The hotline also received calls outside its purpose, including on: gender-based violence, missing or late laboratory results, shortage of commodities at facilities, health care worker workload, health care worker attitudes, changes in health care services (both positive and negative) at facilities and improved quality of life from clients enrolled in the program. Urgent issues received through the hotline were communicated to relevant focal persons in the study (e.g. issues concerning facilities were communicated to the study's clinical coordinator to be addressed with management in relevant facilities).

$\mathrm{CAB}$ members' interactions with community members allowed them to obtain input from the communities which they reported back to the $\mathrm{CAB}$ secretary and study team. These feedback mechanisms allowed the $C A B$ to share the diverse perspectives of a broad range of people in the communities.

I remember in one of the meetings with one of the ministry of health deputy directors she said when we explain about the experiences of the people, it gives them (the Ministry) a clear picture of what is really happening in the facilities. She said boards like $C A B$ should be given more opportunities to voice people's problems because they bring them to surface, things that the Ministry who have had no knowledge of... (CAB member 1).

\section{Value of the $C A B$ to $C A B$ members}

Although the study did not seek to document the reasons why people joined the $\mathrm{CAB}$, the members reported that participating on the $C A B$ had resulted in positive personal outcomes. The outcomes were categorized into: increased learning, increased community recognition and increased ability to help others. The $C A B$ members identified $C A B$ convenings as opportunities to gain knowledge around HIV, research ethics, issues facing the communities where they live and how to work with people from diverse backgrounds. $\mathrm{CAB}$ members also identified areas where they wanted to gain skills and knowledge, and trainings were provided on these topics, including on interpersonal communication and key populations (sex workers and men having sex with men).

$C A B$ members expressed pride in being part of the $\mathrm{CAB}$ and the respect they received from the community, for example when traditional leaders allowed them to communicate about the study in community meetings, as is illustrated in the quote below;

We (the $C A B)$ are taken seriously because when you ask for a slot during meetings, they (community leaders) give you without any problem so they (community leaders) really helped us (the $C A B$ ) in letting the community know about the EAAA, they 
even let us know about other meetings and we would prepare ourselves. (CAB member 7$)$.

$\mathrm{CAB}$ members also took pride in the fact that their work positively affected the lives of others. The majority of $\mathrm{CAB}$ members were already involved in their communities, including as outreach workers and providers of peer support. Through $\mathrm{CAB}$, this involvement extended to encompass treatment education and accompanying people who experienced lapses in treatment back to the clinic. $C A B$ members also mentioned testimonies of people coming back to thank them after they had started treatment and were feeling better. They mentioned providing support to community members with addressing family-related issues. This support was either direct, by providing advice, or indirect whereby they referred the community members to other organizations within their communities who would be able to provide assistance.

It also made us see how important we are as a $C A B$ in Swaziland because of the part we played.

Government alone couldn't have played this role that we play alone... (Focus group participant 3 ).

\section{Challenges in CAB role implementation}

The interviews raised some issues that hindered the CAB's ability to function. The main issues were: lack of adequate funding and dependence of the $\mathrm{CAB}$ on the study team for finances; structure of $\mathrm{CAB}$ meetings; dealing with emotional issues from community members; concerns about $\mathrm{CAB}$ sustainability and lack of community awareness of the CAB's role in some places.

\section{Insufficient financial resources}

$\mathrm{CAB}$ members stated that the budget allocated for $\mathrm{CAB}$ functioning was insufficient. For example, the $C A B$ did not have its own car for site visits, which made it extremely challenging to reach rural clinics in the mountainous Hhohho region, particularly in the rainy season. In terms of communication costs, $\mathrm{CAB}$ members also did not receive paid airtime; despite the creation of a WhatsApp group and a toll-free hotline to contact the $C A B$ secretary, $C A B$ members said they would have benefited from airtime to more easily communicate with each other.

\section{Reliance of the $C A B$ on the study team}

The CAB relied on SWANNEPHA for several aspects of their work including: meeting coordination, $\mathrm{CAB}$ member communication, arranging transportation to visit clinics, travel reimbursements and training logistics. This reliance on the study team was challenging for CAB members who did not live in the geographic areas where the study occurred, as any delays on SWANNEPHA's end in approving logistics and finances impacted the ability of these $\mathrm{CAB}$ members to travel and attend meetings.

\section{$C A B$ structure and functioning}

The $C A B$ members described a positive working relationship with fellow $\mathrm{CAB}$ members. There were logistical concerns mentioned related to the location of the meetings which were held at the SWANNEPHA offices in the city of Mbabane, which resulted in long travel distances to attend meetings. The long travel times affected the length of the $C A B$ meetings, due to the late arrivals of some members.

During the first 18 months of study implementation, $\mathrm{CAB}$ meetings were held on a quarterly basis, where all $\mathrm{CAB}$ members met to discuss issues arising from facility and community visits as well as the hotline. During the $C A B$ case study, it was noted that quarterly meetings were spaced too far apart to allow for the $C A B$ to address critical study implementation issues. The frequency of the meetings was subsequently modified; in addition to the quarterly meetings for the entire $C A B$, bi-monthly meetings were introduced for the $C A B$ chair/co-chair, $C A B$ secretary and one or two $C A B$ members to discuss emerging issues and provide recommendations to the study team. The bi-monthly meetings allowed for a faster response to urgent issues. $C A B$ meetings usually averaged 5 hours, but this was often insufficient to cover all agenda items.

\section{Dealing with emotions and issues beyond the scope of the study}

$\mathrm{CAB}$ members found that people in their communities approached them with a range of issues, some related to the study and others beyond the scope of the study. The study team provided the $C A B$ with a referral list of organizations available in the different study communities, so that $\mathrm{CAB}$ members could make referrals. In the interviews, $C A B$ members mentioned that hearing the issues from community members could sometimes be difficult emotionally, especially since they had a role to provide support to the person confiding in them, as the following quote illustrates;

It happens that at some point the issue becomes very difficult and you can feel it, in a way that you feel like crying, but then you are supposed to be strong for yourself and the client to keep the conversation on progress. (CAB member 8 ).

\section{Lack of awareness in the community}

One $\mathrm{CAB}$ member mentioned that it was challenging to gain the trust of community members without first 
meeting and seeking approval from community leaders. The study's process for introducing CAB members to local communities varied in the beginning; in some places, they met traditional leaders at the Inkhundla (constituency) while in others they met with the inner council. The Inkhundla is an administrative subdivision which includes a cluster of chiefdoms (imiphakatsi) and form the political structures through which Eswatini's administration is organized. This limitation was highlighted in a case report and thereafter, another round of $\mathrm{CAB}$ introductions was done in all the study communities which facilitated better functioning of the $\mathrm{CAB}$.

\section{Discussion}

\section{Promoting human rights and ethical standards}

The importance of engaging communities through establishing $\mathrm{CABs}$ when conducting community-based research has been well articulated in the literature [8-10, 14, 19, 25-27]. CABs have been linked to having a role in protecting the rights of study participants and communities, and in several studies this has meant that they have input in study design and the development of study protocols, and in reviewing informed consent forms and study tools in order to ensure that these initial steps in research design are respectful of participants and communities $[15,19,25]$. During study implementation, the selected $C A B$ members continue to provide input and to address ethical issues $[19,26]$. Since $C A B$ members are usually either selected to represent the diverse demographics in communities or the specific communities being affected by an issue [19], their input during study implementation may help protect the interests of the community.

However, a question arise in terms of how CABs address ethical issues that arise in the community. Is the scope of $C A B$ members limited to discussing ethical issues highlighted by research teams during $C A B$ meetings, or do they have a more independent role in monitoring study implementation to ensure research is implemented according to ethical standards? In the literature, there some accounts of CABs that engage with people in their surrounding areas/communities in the context of sharing information about the study and helping with recruitment and retention $[14,16,19]$ but not in the context of protecting ethical standards or being actively involved in field activities during study implementation. Few studies have described how $C A B$ members extending their scope to practically monitor ethical issues in the community. For example, in a study conducted by Nyirenda et al. [16], CAB members reported accompanying researchers to participant's homes, which was outside the scope of the $C A B$ as defined in their study. Implementation study teams should have reflective consultations during the design phase about the possibilities and mechanisms of granting community representatives a responsibility of monitoring ethical conduct. This includes what internal mechanisms need to be in place so that the $C A B$ is able to affect decisions regarding ethical study procedures and avoid $\mathrm{CAB}$ engagement that is tokenistic, as alluded to by Nyirenda et al. [16].

In the MaxART study, the CAB's role was to promote that the study was conducted in accordance with human rights and research ethical standards. Thus, in addition to representing the diverse demographics during $C A B$ meetings with researchers, the $C A B$ also had a role in being the 'eyes and ears' in communities and in order to provide the study team with insight on its implementation. Having the $\mathrm{CAB}$ actively engage with community members and promote ethical aspects of privacy, consent and confidentiality added value for the research team, since they were able to have access to information from a variety of sources in the community that otherwise could not have been obtained. Some CAB members capitalized on their already established roles in the community to also use these forums to share information about the study and gather insight into people's concerns regarding study implementation. Since they were already trusted members of the community, they were also a trusted link between the study team and community members.

In terms of the role of the $\mathrm{CAB}$ in the communication and education in the community, similar to a study conducted by Lwin et al. [15], the study findings showed that the role of the $C A B$ as understood by $C A B$ members was much broader than originally envisioned by the study team. CAB members took on additional roles as health educators, encouraging better healthcare seeking behaviour and providing healthcare advice to community members. This could be as a result of $C A B$ members merging their $\mathrm{CAB}$ roles with their existing community roles. While this strategy provided a mechanism for the study messages to be shared with communities, it also had potential challenges, especially given that not all the $\mathrm{CAB}$ members had formal training in healthcare or health education. A recommendation as provided by in Lwin et al. would be to capitalize on the opportunity the $\mathrm{CAB}$ poses, and provide appropriate training and support to $C A B$ members and to better combine their $C A B$ role with a range of other community engagement activities [15] .

In this study, we found that the MaxART implementation study did not provide adequate guidance to the $C A B$ about their role of 'protecting communities'. This task was ill-defined in the initial trainings and little guidance was provided by the study team during subsequent trainings about the interpretation and boundaries of 'protection' $\mathrm{CAB}$ members should provide. When forming CABs 
and through-out the implementation of the research, is important that the purpose of the $\mathrm{CAB}$ is clear to all members $[16,27]$ and that the ways that they represent and interact with communities is discussed in order to identify any issues that may mitigate the functioning of the $\mathrm{CAB}$.

\section{Lessons for future CABs in the country and elsewhere}

The main aim in conducting this work was to better understand how the $\mathrm{CAB}$ members perceived their role, to gain insight into the on-the-ground mechanisms that they used to engage with community members and to understand the challenges they faced in their role.

As confirmed in other studies [14, 15, 19, 28], there was value in having a $C A B$ as part of the MaxART EAAA study. CAB members had a role in communicating and sharing information about the study and sometimes dispelled misconceptions about the study in their communities. They provided feedback to the study team; acted as a link to and a voice of the communities; and advocated for the interests of study participants including issues arising around service delivery in health facilities.

While the $\mathrm{CAB}$ contributed to strengthening community engagement, there were some challenges highlighted by $\mathrm{CAB}$ members that provide important lessons for future $C A B$ functioning, including around $C A B$ independence, appropriate budget allocations, increasing psychosocial support for $\mathrm{CAB}$ members, and providing continuous training and capacity building.

The dependence of the CAB on the MaxART study team for support regarding issues such as the logistics of facilitating meetings, especially transport, was a challenge for $C A B$ members. Other studies have recommended the use of non-study-specific CABs [6, 15], arguing that such a structure not only allows for independent functioning of the $\mathrm{CAB}$, but also encourages sustainability as the relationship between the $C A B$ and the community lasts beyond the duration of one study. In our context, a recommendation would be to establish a non-study-specific $\mathrm{CAB}$, the operations and budget of which could be facilitated as an independent entity, or to explore having a $C A B$ that is affiliated with the Ministry of Health, although this would also pose potential issues around independence.

It is essential that $C A B$ members create a safe space for community members to share their concerns and experiences. As a result, some issues arise that are outside the study remit, including community members sharing personal issues. This places a burden on $\mathrm{CAB}$ members emotionally and may require expertise outside of the CAB. It is critical to provide psychosocial support to $\mathrm{CAB}$ members throughout the process. In the MaxART study, a referral list was provided to the $\mathrm{CAB}$, but examining the effectiveness of this referral process was not part of the evaluation. Future studies could evaluate the use of this system.

Training $C A B$ members on topics such as the study protocol, the particularities of the intervention being researched or implemented, and research ethics has been recommended in other studies $[15,16,19]$. Based on the findings of this study, we recommend that rather than only conducting orientation trainings at the beginning of the study, refresher trainings should be offered continuously throughout the period of study implementation. $\mathrm{CAB}$ members should be consulted regarding areas in which they require capacity building and trainings should then be customized. This recommendation has cost implications which should be carefully considered during the $\mathrm{CAB}$ establishment phase.

There are a few accounts in the literature that describe $C A B$ activities in the field and how $C A B$ members engage with people in their surrounding communities. These examples often talk about $\mathrm{CAB}$ members sharing information about the study and helping with recruitment and retention [14, 16, 19], but do not cover the $\mathrm{CAB}$ playing a role in study implementation. In the MaxART study, the CAB's role included being actively involved in advocating for the study to be conducted in accordance with standards of ethical conduct in the study sites, as well as being the eyes and ears in communities and engaging with community members in order to provide the research team with feedback regarding possible ethical malpractices.

When forming CABs, and throughout the implementation of research, it is important that the scope of the $\mathrm{CAB}$ be continuously reviewed with $\mathrm{CAB}$ members $[16$, 27 , and that the ways in which they represent and interact with communities be discussed, in order to identify any issues that may impact the functioning of the $C A B$. $\mathrm{CAB}$ ToRs and functions should be clearly communicated to the $\mathrm{CAB}$, the research team and community members.

\section{Limitations of the study}

This study did not aim to evaluate $\mathrm{CAB}$ functioning in the communities, but rather to understand the roles as perceived by $C A B$ members and the strategies they employed in engaging communities during the MaxART EAAA study. This approach meant that the views of community members and the study team, and their experiences of working with the $C A B$, were not included. In order to gain a holistic understanding of the role of the $\mathrm{CAB}$ in community engagement and its effectiveness, it would be important to also include their perspectives. Furthermore, the inability to interview the the entire $C A B$ membership is a limitation to the study. 


\section{Conclusions}

The experiences of the first $C A B$ members in Eswatini demonstrate that using CABs is a valuable and insightful mechanism to engage communities in a study's implementation. In areas where $\mathrm{CABs}$ are not required for community-based research, governing authorities could use these findings to advocate for the inclusion of community engagement mechanisms, and in particular the creation of CABs, in future research studies. That said, the study also highlights some considerations that could impact $C A B$ functioning. These include the need to clearly define the scope, responsibilities and feedback mechanisms, address issues around independence, provide psychosocial support and offer continuous refresher trainings. These issues should be considered during the early stages of $\mathrm{CAB}$ formation to optimize $\mathrm{CAB}$ functioning.

\section{Abbreviations \\ ARASA: AIDS Rights Alliance of Southern Africa; ART: Antiretroviral therapy; CAB: Community Advisory Board; CAG: Community Advisory Group; EAAA: Early Access to ART for All; FGD: Focus Group Discussion; MaxART: Maximizing ART for Better Health and Zero New Infections; MOH: Ministry of Health; PLHIV: People Living with HIV: \\ SWANNEPHA: Swaziland National Network of People Living with HIV; TOR: Terms of Reference}

\section{Acknowledgements}

We would like to thank all the partners of the MaxART Consortium, the health care workers and community members who made this work possible. We would like to thank Ria Reis for input and advice regarding the study design. We thank Zoe Goldstein and Caitlin Chandler for providing editing services.

\section{Authors' contributions}

$\mathrm{CM}, \mathrm{GK}$, and EV designed the study protocol; CM, GK and BS conducted data analysis in consultation with EV, SJ, RG and EVr. CM drafted the manuscript; $\mathrm{EV}, \mathrm{RG}, \mathrm{EVr}, \mathrm{GK}, \mathrm{SJ}$ and YF critically revised the manuscript for intellectual content. All authors read and approved the final manuscript. CM is the corresponding author for the paper.

\section{Funding}

This work, as part of the MaxART EAAA study, was financially supported by the Dutch Postcode Lottery in the Netherlands, and the Embassy of the Kingdom of the Netherlands in Mozambique. Mylan Laboratories Limited supported the study with donation of the antiretroviral medication Médecins Sans Frontières (with funding from UNITAID) with viral load testing and British Columbia Centre of Excellence in Canada with genotype testing. The funders had no role in study design, data collection, analysis and interpretation or writing the manuscript.

\section{Availability of data and materials}

The datasets used during the current study are available from the corresponding author on reasonable request and with permission of the study's principal investigator.

\section{Ethics approval and consent to participate}

The study received ethical approval from the Swaziland National Health Research Review Board (Ref: MH599C/IRB 0009688/NHRRB 027/16). Signed informed consent was obtained for the semi-structured interviews and verbal consent was obtained from FGD participants after the facilitators explained the rationale for the FGDs. Since the CAB members were used to meeting regularly as a group during $C A B$ meetings, it was felt that verbal consent was suitable for the group discussion as participants were already participating in similar discussions. For individual interviews however, which were inviting participants to reflect on more personal aspects, additional written consent was obtained.

\section{Consent for publication}

Not applicable.

\section{Competing interests}

The authors declare that they have no competing interests.

\section{Author details}

${ }^{1}$ Clinton Health Access Initiative, Mbabane, Swaziland. ${ }^{2}$ Department of Anthropology, Amsterdam Institute for Social Science Research (AISSR), University of Amsterdam, Amsterdam, the Netherlands. ${ }^{3}$ Aidsfonds, Amsterdam, the Netherlands. ${ }^{4}$ Swaziland National Network of People Living with HIV/AIDS, Mbabane, Swaziland. ${ }^{5}$ Global Network of People Living with HIV, Amsterdam, the Netherlands.

Received: 9 November 2018 Accepted: 20 June 2019

Published online: 16 July 2019

\section{References}

1. C. and T. S. A. (CTSA) C. C. E. K. F. Committee. Principles of Community Engagement, NIH Publ. No. 11-7782, https://www.atsdr.cdc.gov/ communityengagement/, 2011.

2. Tindana PO et al. Grand Challenges in Global Health: Community Engagement in Research in Developing Countries. 2007:4(9):1451-1455.

3. Emanuel EJ, Wendler D, Killen J, Grady C. What makes clinical research in developing countries ethical? The benchmarks of ethical research. J Infect Dis. 2004;189(5):930-7.

4. Dickert N, Sugarman J. Ethical goals of community consultation in research. Am J Public Health. 2005:95(7):1123-7.

5. Strauss RP, Sengupta S, Quinn S, Goeppinger J, Spaulding C, Kegeles SM, Millet $\mathrm{G}$. The role of community advisory boards: involving commnunities in the informed consent process. Am J Public Health. 2001;91(12):1938-43.

6. Marsh V, Kamuya D, Rowa Y, Gikonyo C, and Molyneux S. Beginning community engagement at a busy biomedical research programme: experiences from the KEMRI CGMRC-Wellcome Trust research Programme, Kilifi, Kenya. Promot Soc value Res Kenya Examining Pract Asp Collab partnerships using an ethical Framew. 2008:67(5):721-733.

7. Kamuya DM, Marsh V, Kombe FK, Geissler PW, and Molyneux SC. Bioethics low-income settings: selection and perceptions of members of a network of representatives in coastal kenya. 2013;873:255-259.

8. Newman SD, Andrews JO, Magwood GS, Jenkins C, Cox MJ, Williamson DC. Community advisory boards in community-based participatory research: a synthesis of best processes. Prev Chronic Dis. 2011;8(3):A70

9. MacQueen KM, Bhan A, Frohlich J, Holzer J, Sugarman J. Evaluating community engagement in global health research: the need for metrics. BMC Med Ethics. 2015;16(1):44.

10. Nuffield Council on Bioethics. The ethics of research related to healthcare in developing countries. Nuff Counc Bioeth. 2000.

11. J. U. N. P. on H. (Unaids). Ethical Considerations in Biomedical HIV Prevention Trials. 2007.

12. UNAIDS. Good participatory practice guidelines for biomedical HIV prevention trials. Vaccine. 2007:68.

13. Koné A, Sullivan M, Senturia KD, Chrisman NJ, Ciske SJ, and Krieger JW. Improving collaboration between researchers and communities. Public Health Rep. 2000;115(2-3):243-8.

14. Mwinga A and Moodley K. Engaging with community advisory boards (CABs) in Lusaka Zambia: perspectives from the research team and CAB members. BMC Med Ethics. 2015;6(1):1-11.

15. Maung Lwin K, et al. Motivations and perceptions of community advisory boards in the ethics of medical research: the case of the Thai-Myanmar border. BMC Med Ethics. 2014;15(1)

16. D. Nyirenda et al. We are the eyes and ears of researchers and community': understanding the role of community advisory groups in representing researchers and communities in Malawi. Dev World Bioeth. 2017:1-9.

17. Ceasar J, Peters-Lawrence MH, Mitchell V, Powell-Wiley TM. The communication, awareness, relationships and empowerment (C.a.R.E.) model: an effective tool for engaging urban communities in community-based participatory research. Int J Environ Res Public Heal Artic. 2017;14(11):1422. 
18. Safo S, Cunningham C, Beckman A, Haughton L, Starrels JL. A place at the table:' a qualitative analysis of community board members' experiences with academic HIV/AIDS research. BMC Med Res Methodol. 2016;16(1):1-9.

19. Morin SF, Maiorana A, Koester KA, Sheon NM, Richards TA. Community consultation in HIV prevention research: a study of community advisory boards at 6 research sites. J Acquir Immune Defic Syndr. 2003;33(4):513-20.

20. MacQueen KM, et al. What is community? An evidence-based definition for participatory public health. Am J Public Health. 2001;91(12):1929-38.

21. Swaziland hiv incidence measurement survey 2: A population-based hiv impact assessment. 2017:1-4.

22. M. O. F. Health. Swaziland integrated 2015. 2015.

23. Walsh FJ, et al. Impact of early initiation versus national standard of care of antiretroviral therapy in Swaziland's public sector health system: study protocol for a stepped-wedge randomized trial. Trials. 2017;18(1).

24. R. Geut. Practicing community engagement: studying the practice of community engagement in Hhohho region, Swaziland. University of Amsterdam. 2016

25. Quinn SC. Ethics in public health research: protecting human subjects: the role of community advisory boards. Am J Public Health. 2004;94(6):918-22.

26. Campbell MM, et al. Exploring researchers' experiences of working with a researcher-driven, population-specific community advisory board in a south African schizophrenia genomics study. BMC Med Ethics. 2015;16(1):1-9.

27. Shubis K, Juma O, Sharifu R, Burgess B, Abdulla S. Challenges of establishing a community advisory board (CAB) in a low-income, low-resource setting: experiences from Bagamoyo, Tanzania. Heal Res Policy Syst. 2009;7:1-5.

28. Pinto RM, Spector AY, Valera PA. Exploring group dynamics for integrating scientific and experiential knowledge in community advisory boards for HIV research. AIDS Care - Psychol Socio-Medical Asp AIDS/HIV. 2011;23(8):1006-13.

\section{Publisher's Note}

Springer Nature remains neutral with regard to jurisdictional claims in published maps and institutional affiliations.

Ready to submit your research? Choose BMC and benefit from:

- fast, convenient online submission

- thorough peer review by experienced researchers in your field

- rapid publication on acceptance

- support for research data, including large and complex data types

- gold Open Access which fosters wider collaboration and increased citations

- maximum visibility for your research: over $100 \mathrm{M}$ website views per year

At $\mathrm{BMC}$, research is always in progress.

Learn more biomedcentral.com/submissions 